\title{
Desenvolvimento e Utilização de um Programa de Análise de Imagens para o Estudo de Tópicos de Mecânica Clássica
}

Utilization and development of a computer program to perform image analysis for studying topics of classical mechanics

\author{
Vagner B. Barbeta e Issao Yamamoto \\ vbarbeta@cci.fei.br; issaoyam@cci.fei.br \\ Departamento de Física, Centro Universitário UNIFEI \\ Fundação de Ciências Aplicadas \\ Av. Humberto de A. C. Branco, 3972, 09850-901 - S. B. Campo - S.P.
}

Recebido em 1 de abril, 2002. Aceito em 10 de abril, 2002.

\begin{abstract}
Aulas de laboratório são importantes para reforçar conceitos que são introduzidos em aulas teóricas. $\mathrm{O}$ avanço da tecnologia de vídeo e a facilidade em se digitalizar imagens tem permitido o seu uso como instrumento pedagógico em aulas de laboratório. Para o uso de vídeos digitalizados, os experimentos são filmados através de uma câmera VHS e são posteriormente convertidos em um formato-padrão, como o AVI. O vídeo pode ser avançado quadro a quadro e as coordenadas de um determinado ponto podem ser obtidas clicando-se sobre a janela em que o vídeo está sendo reproduzido, permitindo a construção posterior de diversos tipos de gráficos cinemáticos. Do mesmo modo, fotos estroboscópicas podem ser digitalizadas, e as coordenadas de pontos escolhidos podem ser facilmente obtidas. Neste trabalho serão exploradas as possibilidades de uso deste recurso como ferramenta complementar às atividades normalmente desenvolvidas em aulas de física. Serão discutidas também as etapas necessárias para a criação de vídeos digitalizados, bem como será apresentado um software que foi desenvolvido para analisá-los, e que deverá ser utilizado como complemento às atividades de laboratório de Física que são realizadas atualmente pelos alunos do primeiro semestre do UNIFEI. Ao final, serão apresentados alguns exemplos de análises realizadas através do uso do software.
\end{abstract}

Lab classes are very important to emphasize concepts introduced in lectures. The advances of video technology and the simplicity to obtain digital images from analog sources have permitted to apply them as a pedagogical complement in lab classes. In order to use digital videos, the experiments are recorded using a VHS camera and then converted in a standard digital video format, like the AVI. The movie can be played frame by frame, and the coordinates of a given point can be obtained by clicking in the video window, allowing the construction of many kinds of kinematical graphs. In the same way, stroboscopic pictures can be digitized and the coordinates of certain points can be easily obtained. This work is regarding the possibilities of using digital movies and pictures as a complementary activity performed by the students in physics classes. It will be also discussed about the steps that have to be followed to create and to use digital video in the classroom. Finally, it is shown a computer program that was developed at UNIFEI, devoted to analyze digital images (movies and pictures). This computer program is going to be applied in activities that are proposed to freshmen engineering. At the end, some examples of movies and pictures analyzed using the computer program are going to be exploited.

\section{Introdução}

No entendimento de conceitos de cinemática e dinâmica, uma ferramenta poderosa utilizada por educadores é o uso de gráficos. Porém, essa linguagem não é dominada de forma apropriada por muitos es- tudantes. Estes confundem o gráfico da posição em função do tempo com a sua trajetória, ou não distinguem os gráficos de posição, velocidade e aceleração versus tempo, bem como apresentam dificuldades no entendimento do significado das áreas limitadas pelos diversos gráficos, conforme observado por McDermott 
[1] e Beichner [2]. Uma forma de se superar esta dificuldade é fazer com que o estudante possa reconhecer o significado dos diversos gráficos, a partir da coleta de dados e da sua construção, isto é, através de atividades em que participe de forma ativa.

Até há pouco, um dos meios utilizados para a coleta de dados de corpos em movimento baseava-se na foto estroboscópica. Por esse método, utilizava-se uma câmera fotográfica e uma luz estroboscópica para extrair as posições ocupadas por um objeto, através de uma fotografia de exposição múltipla. No entanto, com o avanço da tecnologia de vídeo e da informática, digitalizar e analisar imagens tem se tornado muito popular na última década, o que tem permitido a substituição ou a melhora da antiga técnica da câmera estroboscópica. Esses novos meios apresentam uma série de vantagens como a possibilidade de se obter durante o processo de análise uma rápida visualização dos resultados.

O desenvolvimento das tecnologias para a visualização de vídeos digitais tem possibilitado ao estudante coletar e analisar dados de um experimento, gravados digitalmente, de forma rápida e eficiente, proporcionando assim a oportunidade de se envolver ativamente no processo de aprendizagem [3]. Além disso, atividades que utilizam essas técnicas podem ser desenvolvidas para capacitar os estudantes a investigar a relação entre conceitos físicos relacionados ao movimento e situações que eles podem encontrar fora da sala de aula. Os dados coletados através desse recurso, em geral, passam a fazer mais sentido, deixando de ser apenas um amontoado de números.

Para verificar a eficiência do uso da análise de vídeos como recurso didático, vários estudos foram realizados, como os de Thornton \& Sokoloff [4] e Beichner [5]. Em todos esses trabalhos, observou-se que o recurso era bastante eficaz, e como resultado conseguiram que os estudantes se mostrassem mais motivados, gerando-se mais discussões na sala de aula e diminuindo-se a confusão de alguns conceitos importantes, como a diferença entre os gráficos de velocidade versus tempo e de aceleração versus tempo.

A aplicação das pesquisas educacionais combinadas com o campo da percepção humana pode resultar no desenvolvimento de um material cuja utilização seja excitante para os estudantes e que ao mesmo tempo os ajude a entender conceitos simples, causadores de tanta confusão. Nesse sentido, o uso de novas tecnologias, particularmente do computador, pode contribuir para uma melhora na aquisição e reforço de conceitos por parte do aluno. Deve-se lembrar, no entanto, que o modo como o software é usado na sala de aula é que faz uma grande diferença no impacto educacional que terá. Os professores devem estabelecer formas para que o estudante fique envolvido com o conteúdo, estabele- cendo metas para o aprendizado. É a habilidade no modo de interagir com o conteúdo do vídeo digital que oferece novas e excitantes possibilidades para o seu uso na educação, segundo Williams et al. [6].

Desse modo, uma ferramenta de análise de vídeo digital pode ajudar os estudantes a desenvolver as habilidades para construir e entender gráficos cinemáticos [7], uma fundamental parte da física básica, desde que esteja integrada de forma conveniente com as outras atividades desenvolvidas. É importante ressaltar que com esse tipo de recurso não se pretende eliminar as aulas práticas de laboratório ou reproduzi-las através de simulações. Essa importante ferramenta fornece ao estudante uma ajuda extra no estudo de fenômenos reais, para que os modelos criados para descrevê-los possam fazer mais sentido e para que eles possam ser compreendidos com muito mais clareza.

Neste trabalho discutem-se as etapas necessárias para se criar e utilizar vídeos digitalizados, bem como nossa experiência no desenvolvimento de uma ferramenta que permite realizar a análise de vídeos de sistemas físicos. O programa desenvolvido deverá ser utilizado como complemento às atividades de laboratório que são realizadas atualmente em cursos introdutórios de física do UNIFEI, onde são estudados tópicos de mecânica clássica, bem como de imagens digitalizadas.

\section{Análise de vídeos digitaliza- dos}

Basicamente, são três as etapas no uso de vídeos digitalizados para a realização de experimentos: a primeira é a filmagem do evento que se deseja estudar; a segunda é a digitalização do vídeo; e a terceira é a sua análise através do uso de um software apropriado.

A etapa da filmagem do experimento, em geral, é um processo relativamente simples de ser realizado. Alguns cuidados como o posicionamento da câmera, iluminação adequada, etc., têm que ser tomados, para que a qualidade do resultado final seja satisfatória. Esta é, sem dúvida, a etapa mais simples, porém bastante importante do processo, já que é dela que irão depender em grande parte os resultados obtidos.

A digitalização do vídeo é feita através do uso de interfaces especiais, que permitem transformar o sinal gerado por uma câmera ou videocassete (formato NTSC ou PAL-M), em um formato que seja possível de se reproduzir na tela do computador. Existem alguns formatos diferentes de vídeos digitalizados disponíveis para microcomputadores da linha PC. Os mais comuns são o Quicktime [8] da Apple e o AVI [9] da Microsoft.

O formato Quicktime, desenvolvido pela Apple para ser utilizado originalmente nos microcomputadores da 
linha Macintosh, tem se tornado cada vez mais popular. O lançamento da versão 5 marcou de forma definitiva a sua presença também na linha de microcomputadores PC. Ele tem como uma importante vantagem o fato de manter um controle cuidadoso sobre a taxa de varredura vertical (número de quadros por segundo), o que é fundamental quando se realiza uma análise de vídeo.

No entanto, o formato AVI (Audio Video Interleaved), desenvolvido pela Microsoft, continua ainda sendo o formato mais popular de vídeo digital em microcomputadores. O Windows Media Player é o programa mais utilizado para a execução de vídeos no formato AVI, podendo as versões mais novas reproduzir ainda uma série de outros formatos de áudio e vídeo, como o próprio Quicktime, MP3, etc.

No processo de digitalização de um vídeo, é possível a utilização de uma série de tipos diferentes de compressores de áudio/vídeo, chamados de codecs (COmpressor DECompressor). Os codecs são programas utilizados em conjunto com os programas de reprodução de vídeos digitalizados, e que realizam uma compressão/descompressão do sinal de vídeo para possibilitar uma diminuição do volume de dados gravados no disco. A escolha de um dado tipo de codec depende do tipo de imagem a ser digitalizada, e do quanto se pode comprometer a qualidade dessa imagem, já que a maioria deles realiza uma compressão com perdas. Nesse aspecto, o padrão de compressão MPEG [10], embora permita espaços de armazenamentos significativamente menores para uma boa qualidade de imagem, devido à forma com que realiza a compressão das imagens (compressão temporal, através do uso de quadros principais), não pode ser utilizado para a criação dos vídeos propostos neste trabalho. Na verdade, qualquer tipo de codec baseado em compressão temporal pode levar a resultados ruins quando se realiza a análise do vídeo de um determinado movimento.

Para realizar a terceira etapa, a da análise do vídeo, existem alguns softwares comerciais disponíveis no mercado. Um dos softwares mais completos é o chamado VideoPoint, criado no Dickinson College em Carlisle [11], Estados Unidos, que permite a análise de vídeos que utilizam os formatos Quicktime e AVI. Com esse software, os dados são coletados clicando-se em quadros do filme, nos pontos de interesse, podendo-se combinar esses dados, por exemplo, para obter-se a posição do centro de massa, distância entre pontos, etc. Todos os dados coletados podem ser apresentados de forma tabular ou na forma de gráficos.

O VideoGraph, disponível apenas para computadores da linha Macintosh, foi desenvolvido por R. Beichner da North Carolina State University em 1987/1988 [12]. Essa ferramenta de análise digital de imagens foi criada para uso na fase introdutória de física básica no ensino médio e nas universidades. Ele permite ao estudante assistir movimentos de corpos e usar os recursos gráficos do microcomputador para examinar e analisar esses movimentos. Mais especificamente, o computador reproduz o vídeo na tela enquanto, simultaneamente, cria um gráfico da posição ou velocidade em função do tempo. Uma grande ênfase do VideoGraph é dada para a apresentação simultânea do vídeo com a representação gráfica dos dados coletados. Ele possui ainda um módulo para realizar a captura do vídeo a partir de um videodisco ou de uma placa de captura.

Outro software que tem sido usado por diversas instituições de ensino nos Estados Unidos e no Canadá, para o estudo de vídeos digitalizados, é o Vidshell, um programa gratuito (freeware), desenvolvido por Doyle Davis no início dos anos 90 [13]. Foi desenvolvido utilizando-se uma ferramenta multimídia própria, o ToolBook II, da Asymetrix.

Desenvolvido e distribuído por Robert A. Carlson, o programa World-in-Motion, também é utilizado para a análise de vídeos digitalizados [14]. Uma das características mais interessantes desse software é a extensa base de vídeos disponíveis junto com o programa (mais de 200 vídeos). Além de realizar a análise de vídeos digitalizados, agrega ainda as funções de captura de vídeo, dispensando o uso de outros programas para essa finalidade. Possui ainda um programa tutorial para o aprendizado de gráficos.

O sistema de análise de vídeos digitais via $\mathrm{WWW}$ (World Wide Web), chamado de Digi-Net [15], foi desenvolvido pelo Departamento de Biomecânica da Universidade de Illinois em Urbana-Champaign. Sua principal característica é a de permitir a extração de coordenadas de uma imagem sem usar equipamentos especializados de laboratório. Uma vez coletados os dados on-line, eles podem ser recebidos através de um e-mail. Ele é baseado numa combinação de Java, JavaScript e HTML. Um dos maiores problemas encontrados no Digi-Net é que a ferramenta de extração de coordenadas é muito lenta, isto é, a passagem de um quadro a outro é muito demorada, o que torna a coleta dos dados cansativa. Além disso, não é possível realizar a análise de vídeos além daqueles disponibilizados no site.

Cada um destes softwares destinados à análise de vídeos digitalizados possui uma série de vantagens e desvantagens em relação aos demais. Talvez a principal desvantagem de todos seja o fato de que a adoção de um determinado software exija, muitas vezes, a adaptação das atividades de sala de aula às características do software em uso. A fim de permitir um maior controle sobre essas características, bem como possibilitar a incorporação de funções que eventualmente não estejam disponíveis nos programas existentes, além de garantir um melhor domínio das técnicas utilizadas na criação de 
um software dessa natureza, optou-se pela elaboração de um programa próprio de análise de vídeos digitalizados. A possibilidade de se realizar a análise de fotos estroboscópicas e a de se visualizar uma simulação sobreposta ao evento real são alguns dos diferenciais desse programa que será discutido a seguir.

\section{Desenvolvimento do software de análise de vídeo}

O software que foi desenvolvido [16] permite realizar a análise de vídeos, quadro a quadro, digitalizados no formato AVI, além de permitir também analisar fotos estroboscópicas que tenham sido digitalizadas. Pode-se definir uma origem para o sistema de eixos, e ajustar uma escala de comprimento livre, que permite converter distâncias dadas em pixels para seus valores em metros. Quanto maior for a escala definida, menor é o erro na determinação das coordenadas dos pontos em valores absolutos (isto não influencia na precisão do dado propriamente dito). Com o mouse, retiram-se coordenadas de pontos selecionados que são inseridas numa tabela, à medida que um quadro (frame) é avançado.

É possível, ainda, alterar o sistema de eixos, isto é, determinar uma direção para os eixos (x e y). Assim, podemos facilitar a análise, por exemplo, no experimento de queda livre, no qual se pode colocar a origem no final da régua de medida ou no começo desta. Os dados coletados podem ser exportados para uso posterior em uma planilha eletrônica ou programa gráfico do próprio software, onde os gráficos de posição em função do tempo podem ser criados, e a partir deles, obter-se dados a respeito de velocidade e aceleração em função do tempo, uma vez que o programa gráfico permite o ajuste de uma função linear ou quadrática. Na Fig. 1, apresenta-se uma cópia da tela do programa de análise de vídeo.

O programa desenvolvido possui ainda as seguintes características:

- permite trabalhar com arquivos AVI com diferentes resoluções;

- possibilita a análise de figuras de diferentes resoluções e formatos (GIF, JPG, BMP e WMF);

- permite que sejam analisados vídeos com diferentes taxas de amostragens (o mais comum é que seja utilizado $1 / 30 \mathrm{~s}$, que é o usual das câmeras de vídeo);

- pode-se ampliar a imagem ou reduzi-la para facilitar a coleta de dados;

- qualquer ponto da tela pode ser definido como origem do sistema de eixos;

- pode-se inverter o sentido dos eixos;

- o sistema de eixos pode ser rotacionado de um certo ângulo, para permitir o estudo de movimento sobre planos inclinados, por exemplo;
- os resultados podem ser exportados para um arquivo do tipo texto, ou podem ser colados em uma planilha eletrônica;

- qualquer quadro do vídeo pode ser estabelecido como instante inicial;

- as configurações mais utilizadas podem ser salvas e lidas automaticamente, para reduzir o trabalho de configuração;

- pode-se salvar uma sessão de análise para continuidade posterior;

- é possível visualizar o vídeo ou figura juntamente com a simulação de um modelo criado para representálo;

- é possível deixar rastros dos pontos coletados;

- pode-se avançar ou retroceder para qualquer quadro;

- pode-se medir distâncias e ângulos;

- ao se alterar a escala ou a origem, os pontos coletados são automaticamente recalculados.

Para o desenvolvimento do software foi utilizada a linguagem Visual Basic. A reprodução dos vídeos foi feita utilizando-se chamadas a funções API do Windows que permitem trabalhar diretamente com os dispositivos multimídia. Não foi utilizado o controle OCX do Visual Basic que cuida das funções de multimídia, por este não oferecer os recursos de que necessitávamos, por exemplo, a possibilidade de se reescrever o buffer de vídeo para realizar uma atualização da tela de vídeo.

\section{Uso do software de análise de imagens}

Para se realizar a digitalização de um vídeo, além de uma placa de digitalização, é necessária a utilização de um software de captura de vídeo. A placa de digitalização utilizada foi a miroVideo DC30 plus, e o programa para a captura do vídeo foi o Adobe Premiere 5.0. A taxa de amostragem do software de captura de vídeo foi estabelecida em 30 quadros por segundo, que é exatamente a taxa de varredura da câmera utilizada (na realidade, para os padrões NTSC e PAL-M a taxa de varredura é de 29,97 quadros por segundo).

Estamos, atualmente, buscando substituir esse hardware por quick-cam's (pequenas câmeras para a Internet), que, além do baixo custo (menos de U\$200), são muito mais simples de serem utilizadas. Alguns testes preliminares têm sido feitos com uma câmera Web Cam go Plus da Creative Labs, porém os resultados ainda não são totalmente satisfatórios, já que detectaram-se alguns problemas, como perda de frames a altas taxas e uma aparente imprecisão nos valores obtidos. No entanto, acreditamos que exista grande possibilidade de que possam ser utilizadas com essa finalidade. 
Um problema que se observou logo nos primeiros testes realizados, era o fato de o vídeo obtido apresentar uma imagem "duplicada" quando era filmado um objeto em movimento. Esta imagem duplicada provém da forma de funcionamento de câmeras e de TV's, onde uma tela é composta de duas partes: uma em que são varridas as linhas pares, e a outra em que são varridas as linhas ímpares (também conhecido como varredura entrelaçada). Essa varredura ocorre a uma freqüência de $60 \mathrm{~Hz}$, isto é, num intervalo de tempo de $1 / 60 \mathrm{~s}$ são varridas todas as linhas ímpares, e no intervalo subseqüente de tempo de $1 / 60 \mathrm{~s}$ todas as pares. Desse modo, em $1 / 30 \mathrm{~s}$ são varridas todas as linhas de 1 quadro completo, o que permite obter-se os 30 quadros completos por segundo do padrão de TV utilizado no Brasil. Uma conseqüência deste tipo de técnica de criação de vídeo é o fato de que um objeto que se move rapidamente será composto de duas imagens deslocadas uma da outra, já que cada imagem é amostrada em um instante de tempo diferente (separados por um intervalo de tempo $\Delta \mathrm{t}$ de $1 / 60 \mathrm{~s}$ ). Para evitar esse tipo de dificuldade, a captura de vídeo foi realizada selecionando-se somente um conjunto de linhas (as pares ou as ímpares), de modo que cada tela de vídeo passasse a ter uma resolução, que era metade da resolução daquela permitida pelo software de aquisição, isto é, o vídeo foi digitalizado com uma resolução de 320 × 240 pixels. Este tipo de problema já havia sido reportado anteriormente, por exemplo, no trabalho de Williamson et al. [17], em que se fez o uso de digitalização de vídeo para a análise de um experimento de choque.

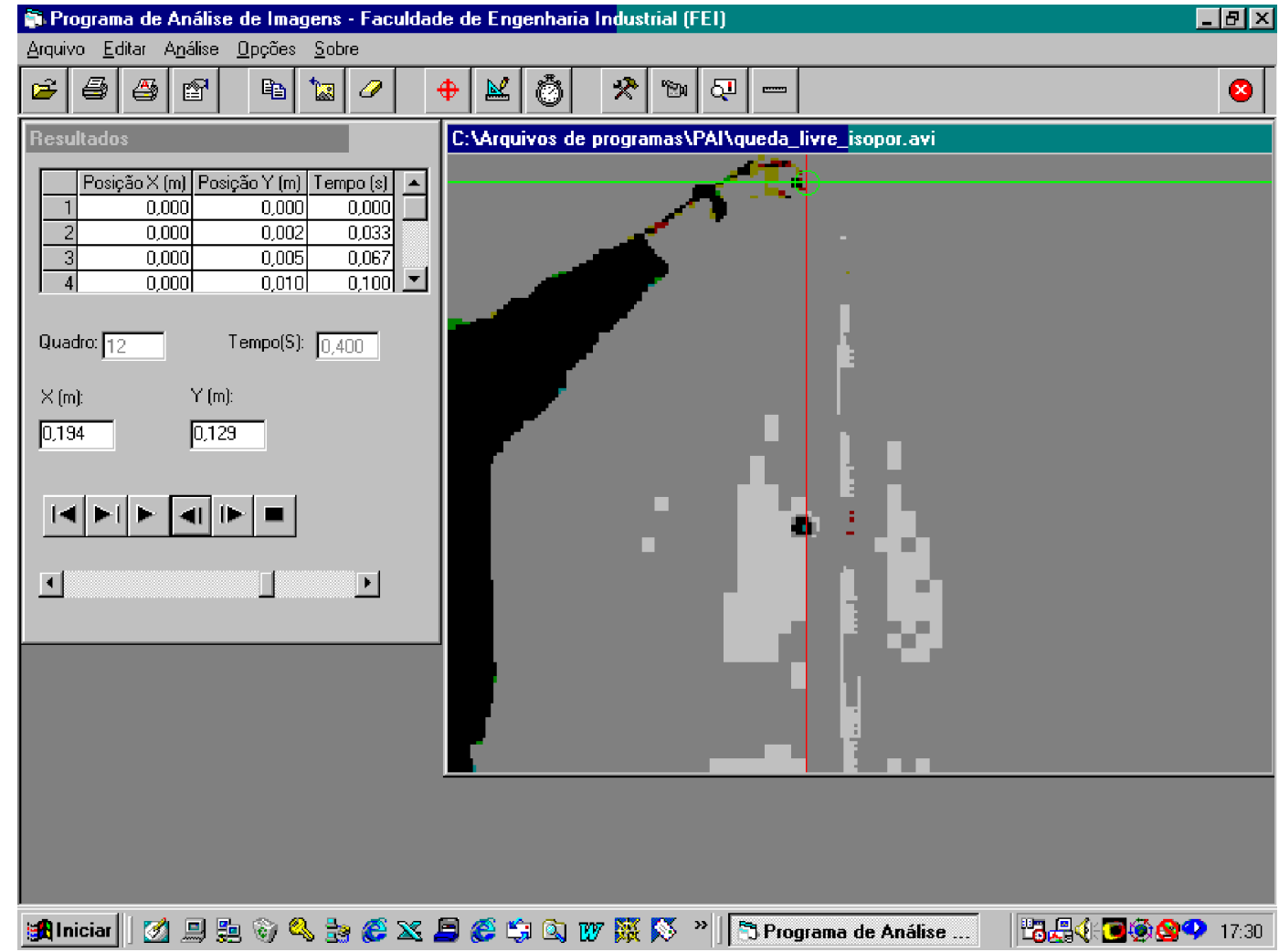

Figura 1. Tela do programa de análise de imagens.

A câmera utilizada para a filmagem foi uma câmera Panasonic NV-M9000, sendo o tempo de abertura do obturador eletrônico ajustado para $1 / 1000 \mathrm{~s}$ (o tempo de abertura do obturador é o tempo em que o dispositivo CCD da câmera está habilitado). Quanto menor o tempo de abertura, mais nítida será a imagem obtida, porém necessita-se, nesse caso, de uma iluminação mais intensa. Um outro problema verificado foi uma oscilação na luminosidade do vídeo, que foi atribuída ao batimento da freqüência de abertura do obturador da câmera com a freqüência da rede $(60 \mathrm{~Hz})$, utilizada para alimentar as lâmpadas fluorescentes da sala onde a filmagem foi realizada. A utilização de lâmpadas de filamento próprias para a iluminação de filmagens fez com que esse efeito desaparecesse. Outro aspecto importante diz respeito à cor do fundo utilizado nas filmagens. Deve-se sempre procurar optar por cores que permitam obter um grande contraste entre a cor do objeto e a cor 
do fundo utilizado. Uma escala colorida foi incluída em todas as filmagens, para tornar possível converter através do software coordenadas dadas em pixels para valores em metros.

Uma vez digitalizadas, as imagens puderam ser então analisadas pelo software desenvolvido. Entre as possibilidades de uso do software, poderíamos destacar as seguintes:

- demonstrações interativas em aulas de teoria: o software pode ser utilizado em aulas teóricas para discutir o movimento de objetos, de modo que, durante o desenvolvimento do tópico, dados possam ser coletados e analisados;

- atividades de laboratório: é possível fazer com que os alunos realizem suas próprias filmagens, digitalizem e a seguir analisem os vídeos produzidos. Em um caso mais simples, cabe ao aluno apenas a análise de um vídeo já digitalizado;

- realização de projetos: é possível fazer com que os estudantes utilizem este tipo de recurso para o estudo em laboratório de sistemas físicos ou o estudo do movimento de objetos reais (o movimento de uma roda gigante, por exemplo).

Descreveremos, a seguir, alguns exemplos de sistemas físicos que foram analisados utilizando-se o software construído, bem como os resultados obtidos com o mesmo.

\section{Exemplos de análises realiza- das com o software}

O sistema foi testado para analisar vídeos como queda livre de um corpo, pêndulo simples, um sistema envolvendo uma polia e um carrinho em um plano horizontal, movimento de um martelo lançado no ar, roda de bicicleta descendo por um plano inclinado, além de algumas fotos estroboscópicas.

Por exemplo, na Fig. 2, mostra-se o vídeo de um carrinho que se desloca horizontalmente, puxado por um bloco que cai verticalmente, através de um fio que passa por uma polia. Pode-se analisar o movimento do carrinho em movimento horizontal ou o do bloco em queda vertical. As diversas posições do bloco em queda estão assinaladas pelos pequenos círculos na janela "Vídeo". A origem do sistema de eixos foi colocada na posição inicial do bloco, orientou-se o eixo $\mathrm{x}$ positivo para a direita e o eixo y crescente para baixo. Escolheu-se uma escala baseada na régua colocada ver- ticalmente, que pode ser vista no lado direito da janela "Vídeo". As coordenadas x e y do carrinho e os respectivos instantes estão registrados na janela "Resultados". Através do menu "Gráficos" do Programa de Análise de Imagens, pode-se observar o gráfico de y versus t, mostrado na Fig. 3. A função quadrática ajustada permite concluir que a aceleração de movimento do carrinho é de aproximadamente $1,01 \mathrm{~m} / \mathrm{s}^{2}$.

Outro exemplo de aplicação do Programa de Análise de Imagens está mostrado na Fig. 4, no vídeo de uma roda de bicicleta que desce através de um plano inclinado [18]. Configurou-se o eixo $\mathrm{x}$ paralelo ao plano inclinado e o eixo y perpendicular a esse plano. A trajetória descrita por um dos pontos da roda pode ser verificada através dos rastros assinalados na janela "Vídeo", e os valores das coordenadas x e y desse ponto estão registrados na janela "Resultados", podendo-se efetuar diferentes estudos sobre esse movimento.

O programa permite fazer uma conexão entre o ponto que está sendo estudado e os dados assinalados na janela "Resultados". Assim, na Figura 4, podemos observar que o ponto assinalado com um pequeno quadrado (apontado com uma seta) na periferia da roda de bicicleta está em contato com o plano inclinado no instante $\mathrm{t}=0,267 \mathrm{~s}$. A Fig. 5 mostra o gráfico da coordenada $\mathrm{x}$ desse ponto em função do tempo. Pode-se verificar através desse gráfico que, no instante $\mathrm{t}=0,267$ $\mathrm{s}$, a reta tangente ao gráfico é praticamente paralela ao eixo dos tempos, ou seja, a componente $\mathrm{x}$ da velocidade desse ponto é praticamente zero (note-se que, por trabalharmos com um intervalo de tempo relativamente grande para esse sistema, não é possível obter com grande precisão o coeficiente angular nulo). Observação semelhante pode ser feita através da Fig. 6, na qual se mostra o gráfico da coordenada y em função do tempo, podendo-se verificar que, no instante $\mathrm{t}=0,267$ s, a coordenada y da velocidade do ponto em estudo é também zero. Isso permite discutir e tornar claro ao aluno que, no caso de rolamento sem escorregamento, o ponto de contato da roda com o plano possui velocidade zero. No caso do estudo de dinâmica de corpo rígido voltado a alunos de engenharia, é comum o uso do conceito de CIR (Centro Instantâneo de Rotação), que é um ponto em torno do qual o corpo rígido como um todo descreve um movimento de rotação. O CIR é um ponto que possui velocidade zero. Assim, é possível visualizar através dessa experiência que o CIR, no caso de uma roda que rola sem escorregamento, é o ponto onde a roda toca o plano. 


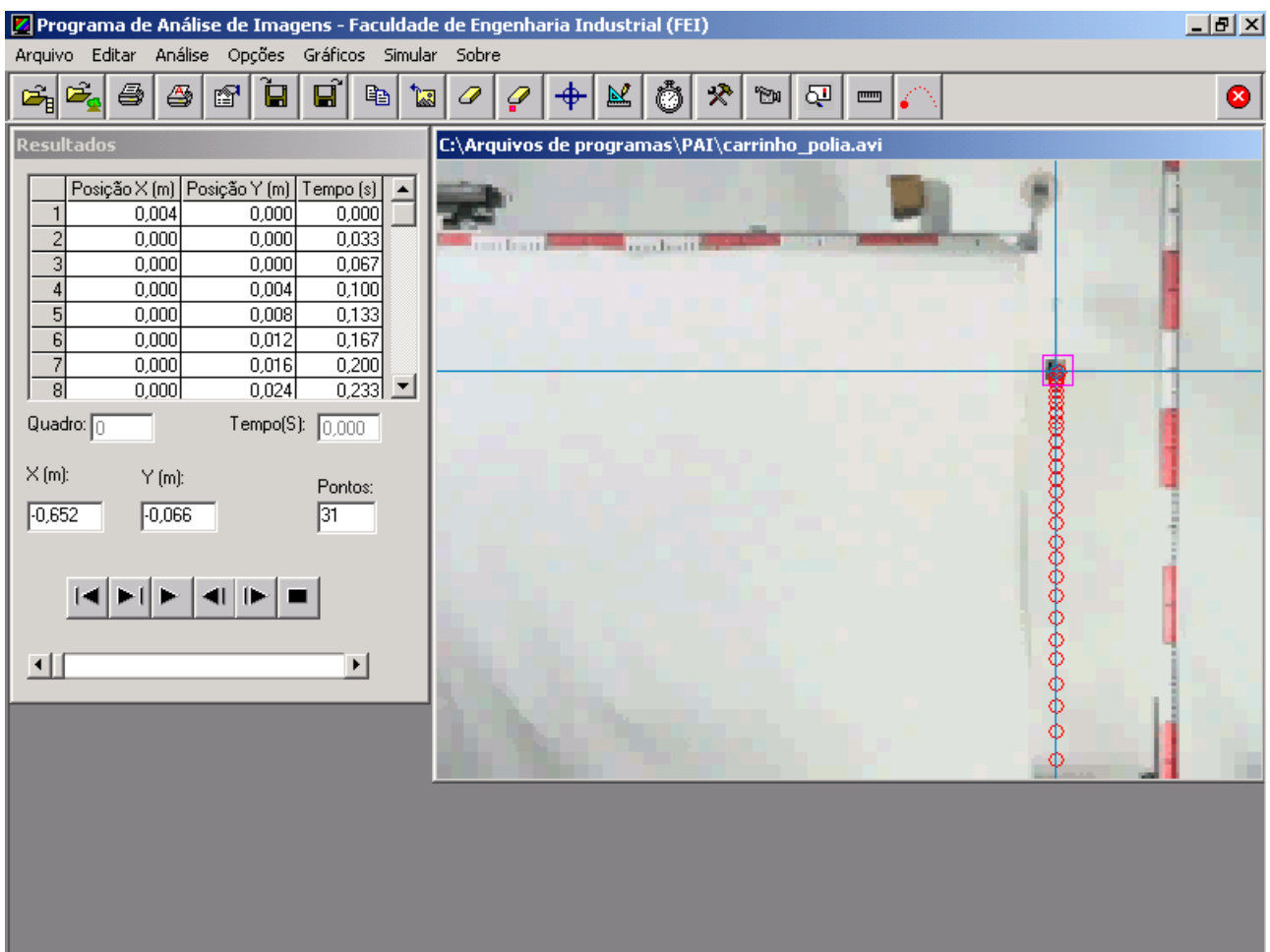

Figura 2. Vídeo do movimento de um carrinho que se move sob a ação de um bloco que cai verticalmente.

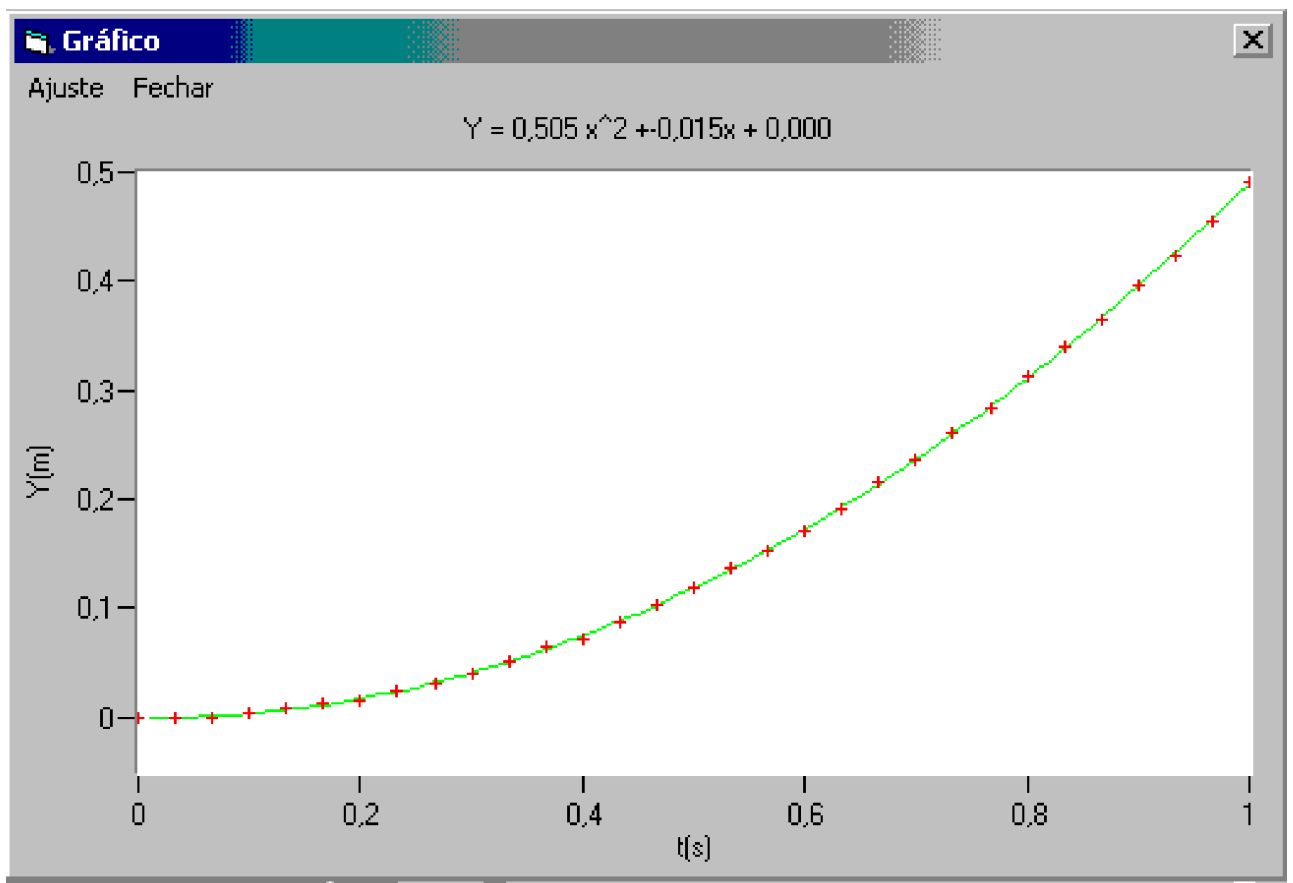

Figura 3. Gráfico da posição y do carrinho em função do tempo e respectiva função quadrática que foi ajustada. 


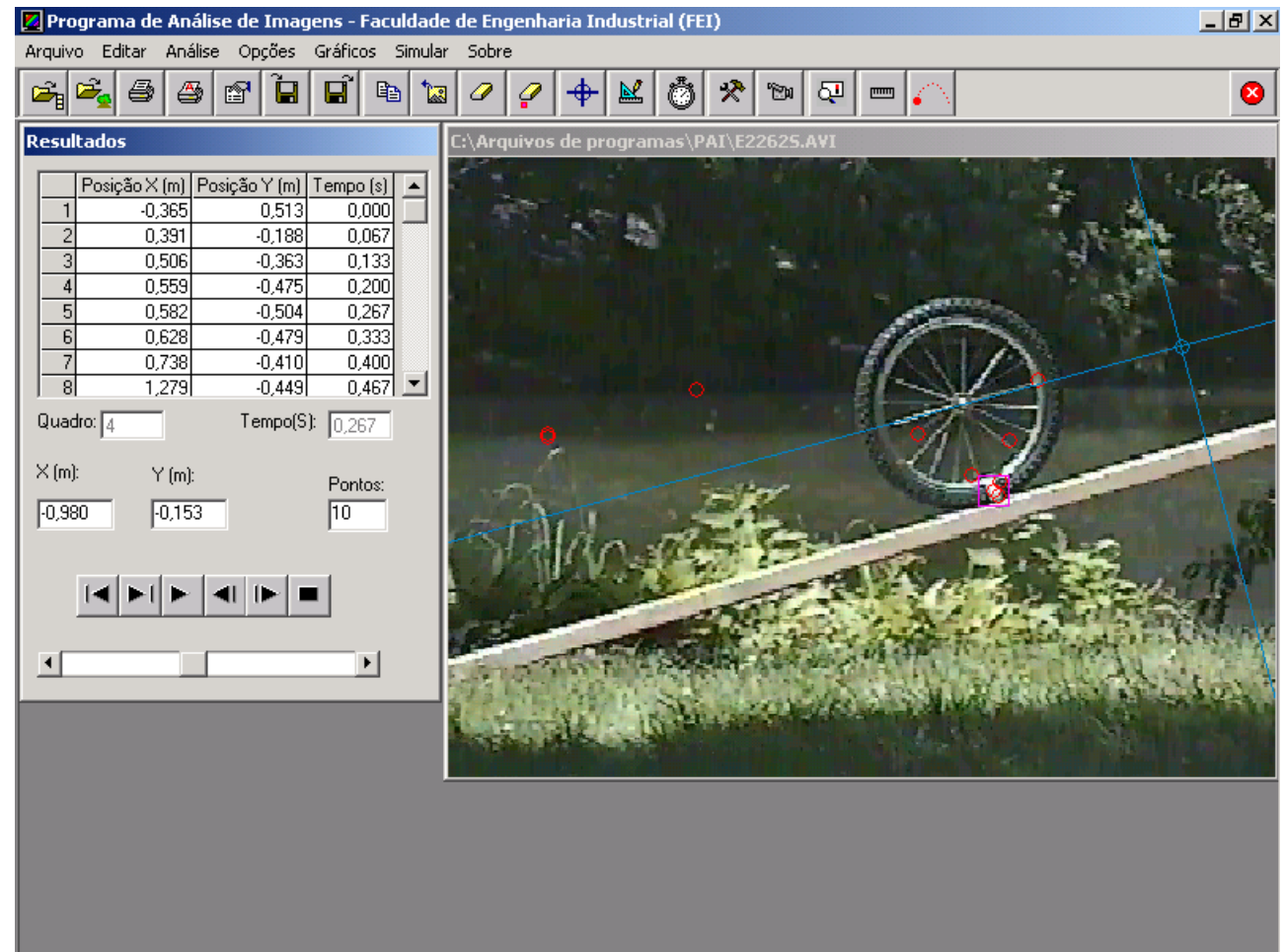

Figura 4. Análise do vídeo de um ponto na periferia de uma roda de bicicleta. A seta indica o ponto discutido no texto.

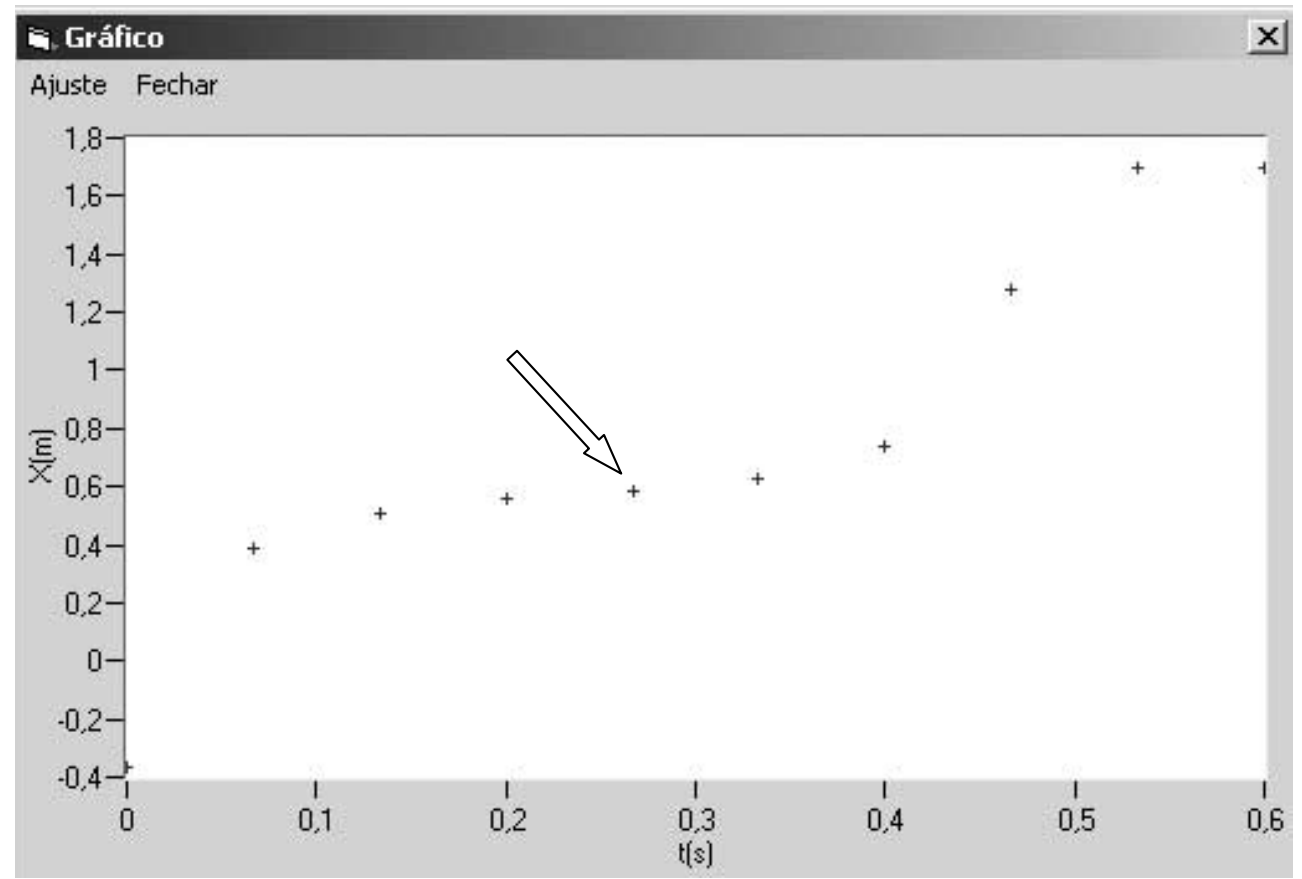

Figura 5. Gráfico da coordenada $\mathrm{x}$ em função do tempo da roda de bicicleta descendo o plano inclinado. A seta indica o ponto para o qual, no instante $\mathrm{t}=0,267 \mathrm{~s}$, a componente $\mathrm{x}$ da velocidade é praticamente nula $\left(\mathrm{v}_{X}=0\right)$. 


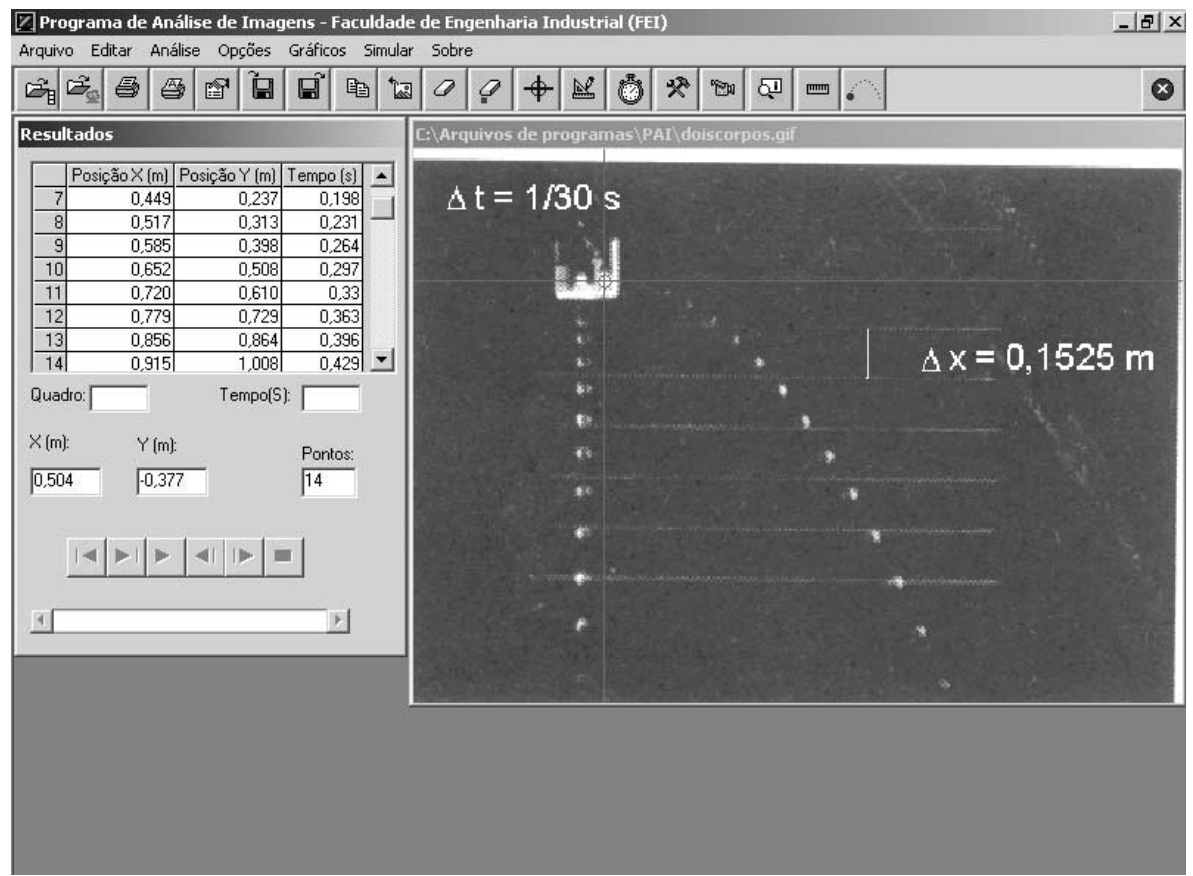

Figura 7. Análise da figura de um projétil lançado horizontalmente.

O Programa de Análise de Imagens foi utilizado para estudar uma foto estroboscópica digitalizada e salva no formato GIF, de um projétil que é lançado horizontalmente no mesmo instante em que outro corpo é abandonado e cai verticalmente, conforme mostrado na Figura 7. Pode-se estudar o movimento do projétil lançado horizontalmente e, a seguir, o movimento do objeto que cai verticalmente. O gráfico y versus t e a respectiva função ajustada, na Figura 8, mostram que o projétil que é lançado horizontalmente se movimenta

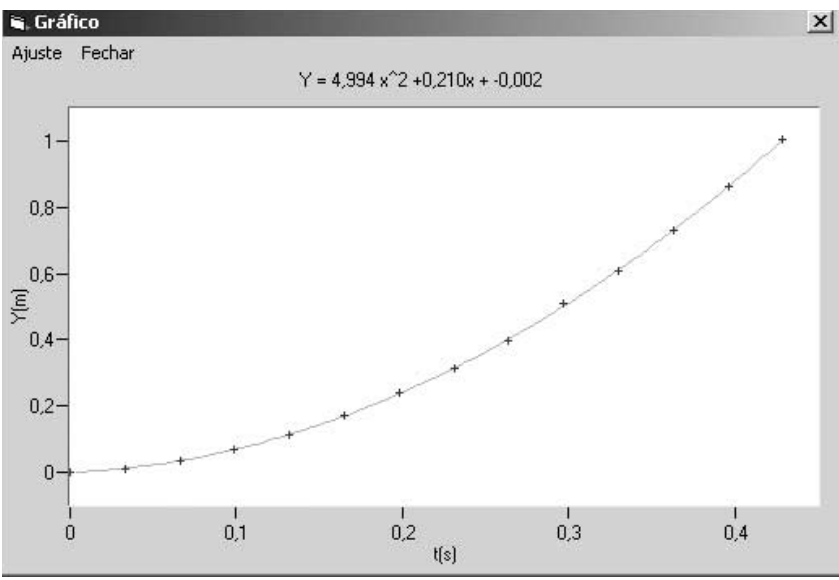

Figura 8. Gráfico y versus t da figura de um projétil lançado horizontalmente.

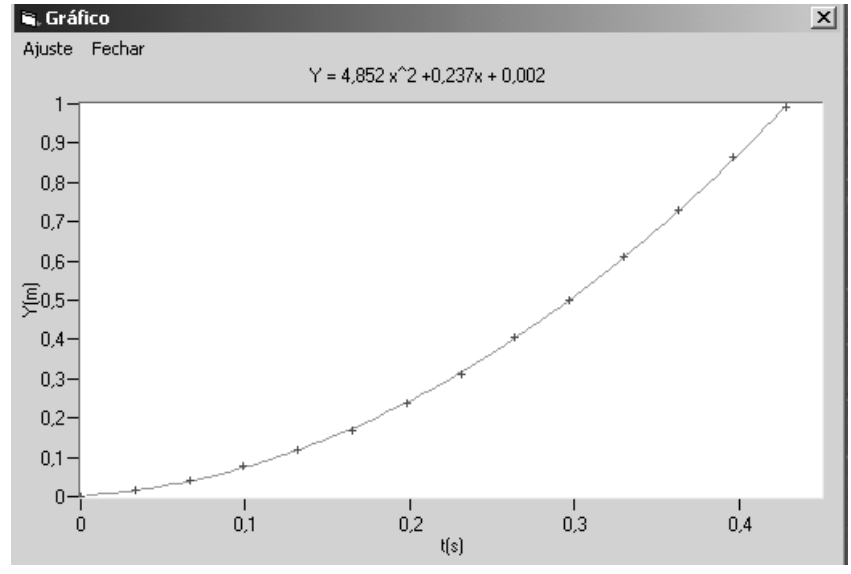

Figura 9. Gráfico y versus t da figura de um projétil abandonado verticalmente.

sob a aceleração vertical cujo valor, obtido experimentalmente, é de $9,99 \mathrm{~m} / \mathrm{s}^{2}$. Verifica-se, através do gráfico y versus t obtido para o corpo que cai verticalmente, na Figura 9, que este movimento se processa sob um valor de aceleração vertical, obtida experimentalmente, de $9,70 \mathrm{~m} / \mathrm{s}^{2}$, notando-se que o erro relativo entre os dois resultados é de cerca de $3,0 \%$.

Diversos outros tipos de estudos poderiam ser feitos nos sistemas discutidos anteriormente. No entanto, o importante é notar que os resultados obtidos com o uso dessa ferramenta são bastante razoáveis e permitem que se faça uma série de discussões com os alunos, além da proposição de uma série de atividades extraclasse. 


\section{Conclusões}

A análise de vídeos digitais é uma forma poderosa de auxílio na interpretação de gráficos cinemáticos. A possibilidade de analisar vídeos de situações reais pode tornar o entendimento desses gráficos mais significativo para os estudantes. É possível, por exemplo, estudar o movimento de uma roda gigante para, a partir daí, obter valores como velocidade linear, velocidade angular, período, etc.

Entre as possibilidades de uso desta ferramenta em laboratório está a análise de vídeos previamente gravados. Em certos casos, pode-se explorar todo o processo de produção desses vídeos, isto é, a filmagem, digitalização e análise, embora isso demande um tempo maior para a sua realização.

No processo de filmagem, é preciso tomar uma série de cuidados, entre eles, o de manter o movimento do objeto num plano que seja normal à direção de filmagem, para evitar erros causados pelos efeitos de perspectiva. É necessário lembrar-se, também, de sempre incluir uma medida-padrão (régua), para que se possam obter os valores de posição em unidades convencionais (metro, $\mathrm{cm}$, etc.).

Os resultados obtidos com o uso do programa de análise de imagens que foi desenvolvido são bastante satisfatórios em termos de erros obtidos entre os valores teóricos e experimentais. A simplicidade de uso, e a possibilidade de inclusão de análise de diferentes tipos de movimento tornam este tipo de ferramenta bastante interessante para o seu uso didático.

Em uma versão futura, o código poderia ser reescrito em Java, de modo a torná-lo multiplataforma, bem como possibilitar a análise de vídeos via Internet. Deverão ser incluídas ainda algumas outras ferramentas de análise, como a obtenção de derivadas e integrais numéricas, o que fará com que as possibilidades de aplicação do software sejam bem mais amplas.

\section{Referências}

[1] L. C. McDermott, M. L. Rosenquist e E. H. van Zee, "Student difficulties in connecting graphs and physics: examples from kinematics", Am. J. Phys., 55, 503-513 (1987).

[2] R. Beichner, "Testing student interpretation of kinematics graphs", Am. J. Phys., 62, 750-762 (1994).

[3] M. Kearney e D. F. Treagust, "Constructivism as a referent in the design and development of a computer program using interactive digital video to enhance learning in physics", Austr. J. of Educ. Techn., 17(1), 64-79 (2001).
[4] R. K. Thornton, e D. R. Sokoloff, "Learning motion concepts using real-time microcomputer-based laboratory tools", Am. J. Phys., 58, 858-867 (1990).

[5] R. J. Beichner, "The effect of simultaneous motion presentation and graph generation in a kinematics lab.", The Phys. Teacher, 27, 803-815 (1990).

[6] J. Williams, A. Lock e C. Burnett. "Digital video for multimedia: considerations for capture, use and delivery". SIMA, Support Initiative for Multimedia Applications, Univ. of Bristol, 1996.

[7] R. J. Beichner, "The impact of video motion analysis on kinematics graph interpretation skills", Am. J. Phys., 64, 1272-1277 (1996).

[8] O programa Quicktime pode ser obtido gratuitamente no endereço http://www.apple.com.

[9] O Windows Media Player, utilizado para tocar vídeos AVI pode ser obtido no endereço http://www.microsoft.com.br.

[10] Informações sobre o padrão MPEG podem ser obtidas, no endereço

http://www.faqs.org/faqs/compressionfaq/part2/section-2.html (acessado em 22/03/00).

[11] Uma descrição mais detalhada do software VideoPoint pode ser encontrada no site:

http://www.lsw.com/videopoint (acessado em 24/07/00).

[12] A página com informações completas do projeto do VideoGraph pode ser acessada pelo endereço http: //www2.ncsu.edu/ncsu/pams/physics/Physics_Ed/ VideoGraph.html (acessado em 01/06/00).

[13] O programa Vidshell, bem como informações complementares sobre ele, podem ser obtidas no endereço http://192.233.237.47/vidshell/vidshell.html (acessado em 23/05/01).

[14] Maiores informações sobre o software World-in-Motion, podem ser obtidas no endereço

http://members.aol.com/raacc/wima.html (acessado em 23/05/01).

[15] O sistema Digi-Net pode ser acessado no endereço http://www.kines.uiuc.edu/digi-net97/Coordination (acessado em 16/05/01).

[16] Uma versão deste software pode ser obtida contatandose diretamente os autores no endereço vbarbeta@cci.fei.br

[17] J. C. Williamson, R. O. Torres-Isea e C. A. Kletzing, "Analyzing linear and angular momentum conservation in digital videos of puck collisions", Am. J. Phys. 68, 841-847, (2000).

[18] Vídeo capturado em 23/05/2001, da World-in-Motion AVI Movie Data Base. 